\title{
An innovative, non-invasive sensation meter allows for a more comprehensive understanding of bladder sensation events: A prospective study in participants with normal bladder function
}

\author{
Hameeda A. Naimi ${ }^{1}$, Anna S. Nagle, PhD², Naomi N. Vinod ${ }^{1}$, Hiren Kolli ${ }^{1}$, Derek Sheen ${ }^{1}$, \\ Stefan G. De Wachter, MD, PhD ${ }^{3}$, John E. Speich, PhD², and Adam P. Klausner, MD ${ }^{1,4}$ \\ ${ }^{1}$ Department of Surgery/Division of Urology, Virginia Commonwealth University School of \\ Medicine, Richmond, Virginia \\ ${ }^{2}$ Department of Mechanical and Nuclear Engineering, Virginia Commonwealth University College \\ of Engineering, Richmond, Virginia \\ ${ }^{3}$ Department of Urology, University Hospital Antwerpen, Edegem, University of Antwerpen, Wilrijk, \\ Belgium \\ ${ }^{4}$ Department of Surgery Hunter Holmes McGuire Veterans Affairs Medical Center, Richmond, \\ Virginia
}

\section{Abstract}

\begin{abstract}
Aims: There is currently no standardized method of characterizing changes in bladder sensation during bladder filling outside of the urodynamics laboratory. The purpose of this investigation was to characterize real-time bladder sensation events using a sensation meter during oral hydration in individuals with normal bladder function.
\end{abstract}

Methods: Participants enrolled in an accelerated hydration study drank 2 L Gatorade-G2® and utilized a sensation meter to record real-time bladder sensation (0-100\%), verbal sensory thresholds, and sensation descriptors of "tense," "pressure," "tingling," "painful," and "other" for two consecutive fill-void cycles.

Results: Data from 21 participants ( 12 females/9 males) were obtained and demonstrated an average of 8-9 sensation events (significant changes in sensation) per fill with no differences in the total number of sensation events and volume between sensation events (fill 1 vs fill 2). An increased number of sensation events occurred at higher capacity quartiles. Event descriptors of "pressure" and "tingling" were the most commonly chosen descriptors in both fills.

Conclusions: The innovative sensation meter includes the sensation event descriptors of "tense," "tingling," "pressure," and "painful," to enable a more comprehensive understanding of

\footnotetext{
Correspondence: Adam P. Klausner, Division of Urology, VCU Medical Center, Box 980118, Richmond, VA 23298-0118. apklausner@vcu.edu. 
bladder sensation as well as real-time identification, quantification, and characterization of sensation events. The study demonstrates 8-9 events per fill, acceleration of sensation during filling, and unique sensation event descriptor patterns. This technology may be helpful in the identification of novel sensation patterns associated with overactive bladder (OAB) and aging.

\section{Keywords}

diuresis/physiology; sensation/physiology; urinary bladder/physiology

\section{1 | INTRODUCTION}

Overactive bladder (OAB), as defined by the International Continence Society (ICS) is "a syndrome characterized by symptoms of urgency, with or without urgency incontinence, usually with increased daytime frequency and nocturia." 1 Nearly 30 million adults over the age of 40 years in the US display "bothersome symptoms of OAB." 2 While estimates of health care burden vary, it is generally accepted that patients who are more symptomatic report poorer quality of life and experience worse mental health outcomes. ${ }^{3,4}$ Furthermore, because the only absolute term in the ICS definition is "urgency," OAB can be viewed as a disorder of bladder sensation.

Currently, there is no objective means to characterize bladder sensation outside of the urodynamics (UDS) laboratory. Validated surveys and void diaries give some information about bladder sensation and urgency; however, these instruments are subject to significant recall bias and do not assess real-time sensation. ${ }^{5}$ Therefore, the development of an instrument to objectively measure bladder sensation outside of the UDS laboratory represents a critical research objective. Furthermore, this technology must be able to provide objective information at variable fill rates to be robust in the office or home settings.

In order to improve our understanding and diagnosis of bladder sensation, a previously engineered sensation meter was developed to record real-time bladder sensation and compare data to ICS verbal sensory thresholds (VST) of first sensation (FS), first desire (FD), and strong desire (SD) during hydration and urodynamic studies. ${ }^{6}$ This resulted in the generation of sensation-capacity curves which showed differing patterns between normal individuals and those with $\mathrm{OAB}$ as well as differing responses to slower or faster filling. ${ }^{6}$

Focus-group introspection studies of bladder sensation in both healthy volunteers and OAB patients examined individual changes in sensation that occur during filling. ${ }^{7,8}$ The most common reported changes identified during these studies were labeled with unique descriptors and included the terms "pressure," "tingling," "tense," and "painful."7,8 This background work provides the rationale for the inclusion of four sensation descriptors (pressure, tingling, tense, painful) in our sensation meter which can evaluate real-time changes in sensation hereafter called "sensation events."

Therefore, the purpose of the current study was to utilize our sensation meter to more objectively identify, quantify, and characterize individual sensation events employing a variable fill rate protocol. Because data from previous bladder sensation studies demonstrate that "patients with $\mathrm{OAB}$ complaints experience and describe bladder sensation in the same 
way as healthy volunteers," we have chosen to first test our new sensation meter on healthy volunteers without any significant urinary urgency. ${ }^{8}$ The goal of this study, was to test new sensation technology on normal participants that could ultimately be used for comparison studies in the non-invasive evaluation and diagnosis of OAB.

\section{2 | MATERIALS AND METHODS}

A power study, performed in $\mathrm{R}$ ( $\mathrm{R}$ Development Core Team, Vienna Austria) using the data published by Heeringa et $\mathrm{al}^{7}$ comparing bladder volumes of young, asymptomatic volunteers at the sensation of weak awareness (analogous to FS) to weak desire (analogous to FD), yielded a sample size of 17 (16.4) participants required to detect a difference with a power level of 0.80 . Anticipating that up to a third of participants may fail to complete the protocol, 24 participants (13 females/11 males) without symptoms of urinary urgency were recruited for this accelerated oral hydration study. Twelve (50\%) of the participants self-identified as Asian, eight (33\%) identified as Caucasian, three (12.5\%) identified as African American, and one $(4.17 \%)$ identified as Hispanic. All individuals had been previously trained on the sensation meter using a standardized training video. Volunteers completed an ICIq-OAB survey and qualified to participate if they scored 0 (never) on question 5a ("Do you have to rush to the toilet to urinate?") and no more than one on the other survey questions. Age, gender, race, body mass index (BMI), medical history, prescription medications as well as food and beverage intake for that day were recorded. ${ }^{10}$

\section{1| Sensation meter}

The sensation meter is a graphical user interface implemented using LabView (National Instruments, Austin, TX) software on a tablet touch-screen Surface Pro3 (Microsoft, Redmond, WA). It permits each participant to quantify his or her bladder sensation from $0 \%$ (feeling of complete bladder emptiness) to $100 \%$ (feeling of complete bladder fullness) every time a change in sensation is perceived (Figure 1A). ${ }^{6}$ Participants recorded ICSdefined standard VSTs (Figure 1A, right), and with each sensation change, individuals were trained to mark one or more sensation descriptors: tense, pressure, tingling, painful, and other (Figure 1B, left). Participants were not prompted to use the sensation meter throughout the duration of the study. At the end of the study, individuals completed surveys on difficulty of use of the sensation meter and understanding instructions, both of which were assessed on 10-point Likert scales.

\subsection{Accelerated hydration}

Upon presentation, participants voided and post-void residual (PVR) volume was measured using BladderScan (Verathon, Seattle Washington). The Sensation Meter was then started and participants were instructed to drink 2 L G2-Gatorade ${ }^{\circledR}$ as rapidly as possible while seated comfortably with the meter positioned on a Mayo stand in front of their chairs. When participants reached $100 \%$ sensation, the first fill was concluded. At this point, participants were escorted to the bathroom and instructed to void in a graduated collection device and PVR volumes were again measured with BladderScan. Total filling volume was defined as the voided volume plus the PVR. At this point, participants returned to the testing chair, and the sensation meter was reset to initiate the second fill. No additional hydration was 
permitted, and the identical protocol was repeated. Therefore, each participant used the sensation meter through two complete fill-void cycles.

\subsection{Sensation events, descriptors, and VSTs}

The following data were collected during two fill-void cycles: voided volumes, PVR, and duration of filling. In addition, sensation meter data including bladder sensation (0-100\%), VSTs, and individual sensation event descriptors were collected. Example participant data is shown in Figures 1C and 1D. To be counted as an independent sensation event, the sensation meter needed to be moved to a new sensation level and left there for $>10 \mathrm{~s}$. This method was employed to allow participants to achieve new steady sensation levels. A MATLAB R2017a code was used to automatically implement this algorithm. In order to estimate volume and percent capacity at each sensation event, we determined an average within-fill rate by dividing total volume (voided volume + PVR) by the total fill duration separately for fill 1 and fill 2 as described in our prior study. ${ }^{6}$

\section{4| Statistical analyses}

All statistics were computed using Prism v7.03 (GraphPad Software, La Jolla, California). D'Agostino-Pearson tests were used to determine distribution normality. Bivariate comparisons were performed with Student's t-test and Wilcoxon test when appropriate. Multivariate comparisons were performed with repeated measures ANOVA with multiple comparison post-tests using Dunn's for paired data and Sidak's for unpaired data to identify differences between chosen data sets. All data are reported as mean \pm standard error.

\section{3 | RESULTS}

Twenty-one participants completed the protocol. Participant characteristics included mean age of $24.5 \pm 1.1$ years and mean BMI of $24.3 \pm 1.1$. All participants were healthy and not on any medications known to affect bladder function. Participants reported ease of usage and device understanding on a Likert scale of 0-10 (easy to difficult) as $0.05 \pm .0 .05$ on both items. Sensation event characteristics are displayed in Figure 2. In all box plots (Figures 24), the bottom and top lines indicate min/max, the bottom and top box borders indicate 25th/ 75th quartiles, and the middle line represents median. Mean is indicated with a " + " sign only if different from median by $>10 \%$. Average number of sensation events did not differ between fills (fill $1=9$, fill $2=8$ ), (Figure 2A). This suggests relative consistency of sensation events, despite variable fill rates. However, duration between sensation events decreased in fill $2(P<0.0001$, Figure 2B), likely due to faster filling. There was no significant difference in average volume per sensation event between fills (Figure 2C).

To compare frequency of sensation events at different stages of filling, sensation events for both fills were compared across capacity quartiles and as histograms (Figures 3A and 3B). This demonstrates an increased number of sensation events with increased filling or an acceleration of sensation. The data also show that frequency of sensation events increases exponentially in fill 1 and linearly in fill 2, likely reflecting the variable fill rate. As a group, the number of sensation events in the first half of filling (capacities $<50 \%$ ) was 65 in fill 1 and 59 in fill 2, and the number of sensation events in the second half of filling (capacities 
$250 \%$ ) was 127 in fill 1 and 114 in fill 2 . This approximate doubling of sensation events represented a significant change in sensation event frequency between the two halves of filling $(P<0.05)$. Together, this data reveals that the majority of bladder sensation events, regardless of fill rate or end volume, are perceived at $>50 \%$ bladder capacity (Figures $3 \mathrm{~A}$ and 3B).

Sensation event descriptors (tense, pressure, tingling, painful) are displayed in Figure 4. Three participants (all females) were excluded from the descriptor analysis as they marked no descriptors. The descriptor other is not included as it was never selected. Regardless of fill, pressure and tingling were felt throughout filling while tense was only felt at sensations $>40 \%$ and painful was only rarely felt at sensations $>75 \%$. Descriptor combinations such as the frequency of pressure alone versus pressure-tingling were explored; however, no one pattern dominated among individuals, further illustrating the complexity of sensation description.

The majority of participants rated the sensation meter as easy to use (rating $=0 / 10$ ) and easy to understand (rating $=0 / 10$ ). On average, however, individuals only selected descriptors with every perceived sensation about $75 \%$ of the time in both fills.

\section{4 | DISCUSSION}

This study demonstrates the utility of a bladder sensation meter to generate continuous realtime sensation data during filling as well as to fully characterize unique changes in sensation that we call "sensation events." A key finding from this variable fill rate investigation was the identification of a relatively consistent number of sensation events within each fill (approximately 8-9 events). Furthermore, we found that the majority of bladder sensation events, regardless of fill rate or end volume, are perceived at $>50 \%$ bladder capacity, demonstrating that sensation accelerates over the course of filling. Finally, our characterization of sensation events using terminology from previously published focus group introspection studies, demonstrates that pressure and tingling are the most common descriptors throughout filling, with tense and painful occurring less commonly and only at higher capacities. ${ }^{7,8}$ The benefit of this variable fill rate protocol is that unlike previous studies performed at constant maximal diuresis, variable rates may more closely approximate real-life conditions and thus perhaps be more clinically relevant. ${ }^{7,8}$

Previous studies have utilized bladder diaries, urge scales, bladder questionnaires, and focus groups to study bladder sensation. However, these methods rarely reflect real-time sensation as individuals must recall past events. ${ }^{5}$ Analysis of over 600 articles assessing urinary urgency by Das et $\mathrm{al}^{11}$ reported that sensory quality (descriptors) was the second least common examined dimension of sensation, illustrating the potential for better understanding of perceived bladder sensation and associated description. This highlights the importance of new technologies, such as our sensation meter, which have the potential to vastly improve sensation diagnostics not only through the generation of unique sensation-capacity curves, but also through identification, quantification, and characterization of individual sensation events. 
Previously conducted hydration studies have demonstrated the limited value of episodic VSTs as a sole means of characterizing real-time bladder sensation. ${ }^{6}$ Indeed, perception of bladder fullness was not limited to three events in the current study. Rather, sensation events averaged nine in fill 1 and eight in fill 2, suggesting that three VSTs are insufficient to fully characterize sensation. Analysis of change in number of sensation events (not shown) revealed that most individuals had $<25 \%$ change in the number of sensation events, regardless of fill rate. The minority of individuals with $>25 \%$ change in number of sensation events (mainly a reduction in number) suggests that these individuals may have falsely perceived abdominal distension as bladder sensation in the initial fill or that their bladders became less sensitive with subsequent filling. The latter has been demonstrated experimentally through cystometry studies in rats and cats, where higher filling rates were associated with decreased afferent activity. ${ }^{12,13}$

Studies by Robertson et al, ${ }^{14}$ revealed that supra-physiologic fill rates, such as those performed in UD studies, gave rise to higher end volumes. ICS defines the supra-physiologic filling as rates greater than predicted maximum body weight $(\mathrm{kg}) / 4$ expressed as $\mathrm{mL} / \mathrm{min}$. ${ }^{1}$ Using this definition, physiological filling was seen in 20/21 (95\%) of individuals in fill 1 but only 11/21 (52\%) individuals in fill 2 in the current investigation. This is likely from a ramp-up of bladder diuresis during fill 1 and maintaining a more constant rate in fill 2 . This data is consistent with our previous ultrasound study results and supports our variable fill rate protocol design. ${ }^{6}$ The relative consistency of data, despite dramatic changes in fill rates in the current investigation, demonstrates the versatility of our sensation meter, which can be used at variable fill rates in normal filling, hydration studies, and during urodynamics.

Studies attempting to understand sensation origin and mechanism have focused on afferent fiber activity and diversity. Gillespie et al ${ }^{15}$ described bladder sensation in the context of continuous afferent output to the CNS, suggesting that through unknown pathways, higher brain centers generate sensation in response to only a select set of afferent fibers. Participants in this study, perceived sensations at every quartile capacity, showing that bladder sensation is continuous rather than episodic and that participants became aware of their bladders at a spectrum of capacities. Comparison between void diaries and focused introspection studies by De Wachter et al ${ }^{16}$ noted that when participants record perceived sensation right before voiding (void diaries), they generally mark higher than when they record sensation over the course of filling. To a certain extent, both De Wachter and Gillespie associate bladder fullness and readiness to void with modulation of centrallyperceived afferent signaling. ${ }^{15,16}$ De Wachter et al ${ }^{16}$ also concluded that centrally-motivated deviations in pre-void behaviors may influence interpretation of peripheral afferent input. Because afferent signaling pathways may be associated with fine-tuning of bladder sensation, this suggests that individual sensation may be perceived to a greater extent towards the end of bladder filling. Our current data, demonstrating an acceleration of sensation at higher capacities, supports this paradigm.

In terms of individual event descriptors, De Wachter et $\mathrm{al}^{16}$ reported that most individuals experience pressure as a continuous, intensifying sensation while tingling comes and goes and is not necessarily experienced by all participants. ${ }^{7,8}$ In a study by Das et al, ${ }^{17}$ individuals with and without $\mathrm{OAB}$ reported sensation qualitatively through questionnaires. 
Selection of pressure was more associated with healthy individuals and tingling was removed from consideration after $>75 \%$ failed to select it. ${ }^{17}$ Results from our current hydration study showed that more than half of all perceived pressure and tingling descriptors were recorded at $>50 \%$ sensation, suggesting that pressure may not be as continuously perceived as previously reported. ${ }^{7,8}$ Curiously, in our study, tense was selected more often in the second fill (44 vs 26 selections), whereas tingling was selected less often (65 vs 90 selections). This may reflect a generally higher likelihood of feeling tense with faster filling. Conversely, the sensation of tingling might be more reflective of slower filling or be associated with earlier, ambiguous bladder sensations such as abdominal distension.

Limitations of this study include the young age of the participants as well as the lack of inclusion of individuals with well-characterized voiding dysfunction such as OAB. However, the goal of this initial study was to characterize sensation events in normal, healthy volunteers who were young enough to be unlikely to have subclinical bladder problems. We plan to perform future studies evaluating the sensation meter in more diverse populations and will use these initial results for comparison. This study demonstrated that the sensation meter is easy to use and can be employed during variable fill rates. This non-invasive sensation meter may potentially enable improved phenotyping of OAB and other forms of voiding dysfunction.

\title{
5| CONCLUSIONS
}

This study demonstrates that normal healthy individuals generally perceive 8-9 unique sensation events during filling even at variable rates. In addition, results from this investigation demonstrate that sensation accelerates during filling and that unique sensation patterns can be identified through the use of sensation event descriptors (tense, tingling, pressure, and painful). Our non-invasive sensation meter allows for a more comprehensive understanding of bladder sensation events. This novel technology may be useful in the identification of novel sensation patterns associated $\mathrm{OAB}$ and other forms of voiding dysfunction in office and home settings.

\section{ACKNOWLEDGMENTS}

The authors would like to thank Paul Ratz, R. Wayne Barbee, Kimberly Bradley, Zachary Cullingsworth, Randy Vince, Uzoma Anele, Jary Varghese, Sandy Smith, and Jacqueline Morin for their technical contributions to this work. This work was supported by grants from the National Institutes of Health (R01DK101719).

Funding information

Virginia Commonwealth University School of Medicine Summer Research Fellowship Program; National Institute of Diabetes and Digestive and Kidney Diseases,Grant number: R01-DK101719

\author{
Abbreviations: \\ ICS International Continence Society \\ OAB overactive bladder \\ PVR post-void residual
}



UDS
urodynamics
VST
verbal sensory thresholds

\section{REFERENCES}

1. Abrams P, Cardozo L, Fall M, et al. The standardisation of terminology of lower urinary tract function: report from the standardisation sub-committee of the International Continence Society. Neurourol Urodyn. 2002;21:167-178. [PubMed: 11857671]

2. Coyne KS, Sexton CC, Vats V, Thompson C, Kopp ZS, Milsom I. National community prevalence of overactive bladder in the United States stratified by sex and age. Urology. 2011;77:1081-1087. [PubMed: 21256571]

3. Coyne KS, Kvasz M, Ireland AM, Milsom I, Kopp ZS, Chapple CR. Urinary incontinence and its relationship to mental health and health-related quality of life in men and women in Sweden, the United Kingdom, and the United States. Eur Urol. 2012;61:88-95. [PubMed: 21831517]

4. Lai HH, Vetter J, Jain S, Andriole GL. Systemic nonurological symptoms in patients with overactive bladder. J Urol. 2016;196: 467-472. [PubMed: 26997309]

5. Bright E, Drake MJ, Abrams P. Urinary diaries: evidence for the development and validation of diary content, format, and duration. Neurourol Urodyn. 2011;30:348-352. [PubMed: 21284023]

6. Nagle AS, Speich JE, De Wachter SG, et al. Non-invasive characterization of real-time bladder sensation using accelerated hydration and a novel sensation meter: an initial experience. Neurourol Urodyn. 2017;36:1417-1426. [PubMed: 27654469]

7. Heeringa R, de Wachter SG, van Kerrebroeck PE, van Koeveringe GA. Normal bladder sensations in healthy volunteers: a focus group investigation. Neurourol Urodyn. 2011;30:1350-1355. [PubMed: 21608021]

8. Heeringa R, van Koeveringe GA, Winkens B, van Kerrebroeck PE, de Wachter SG. Do patients with $\mathrm{OAB}$ experience bladder sensations in the same way as healthy volunteers? A focus group investigation. Neurourol Urodyn. 2012;31:521-525. [PubMed: 22396435]

9. Speich JE, Nagle AS, De Wachter SG, Klausner AP. The sensation meter: an unprompted method to characterize patient-reported bladder sensation in real-time. American Society of Mechanical Engineers (ASME). Frontiers in Biomedical Devices, 2017 Design of Medical Devices Conference: V001T04A001. 10.1115/DMD2017-3433. Available at: http:// proceedings.asmedigitalcollection.asme.org/proceeding.aspx? articleid=2661331

10. Abrams P, Avery K, Gardener N, Donovan J, ICIQ advisory board: the international consultation on incontinence modular questionnaire: www.iciq.net. J Urol. 2006;175:1063-1063. [PubMed: 16469618]

11. Das R, Buckley J, Williams M. Dimensions of sensation assessed in urinary urgency: a systematic review. J Urol. 2013;190: 1165-1172. [PubMed: 23643596]

12. De Wachter S, De Laet K, Wyndaele JJ. Does the cystometric filling rate affect the afferent bladder response pattern? A study on single fibre pelvic nerve afferents in the rat urinary bladder. Neurourol Urodyn. 2006;25:162-167. [PubMed: 16372317]

13. Satchell P, Vaughan C. Bladder wall tension and mechanoreceptor discharge. Pflugers Arch. 1994;426:304-309. [PubMed: 8183641]

14. Robertson AS, Griffiths CJ, Ramsden PD, Neal DE. Bladder function in healthy volunteers: ambulatory monitoring and conventional urodynamic studies. Br J Urol. 1994;73:242-249. [PubMed: 8162500]

15. Gillespie JI, van Koeveringe GA, de Wachter SG, de Vente J. On the origins of the sensory output from the bladder: the concept of afferent noise. BJU Int. 2009;103:1324-1333. [PubMed: 19344428]

16. De Wachter SG, Heeringa R, van Koeveringe GA, Gillespie JI. On the nature of bladder sensation: the concept of sensory modulation. Neurourol Urodyn. 2011;30:1220-1226. [PubMed: 21661031]

17. Das R, Buckley J, Williams M. Assessing multiple dimensions of urgency sensation: the university of south Australia urinary sensation assessment (USA2). Neurourol Urodyn. 2017;36:667-672. [PubMed: 26999753] 
(A)

(C)

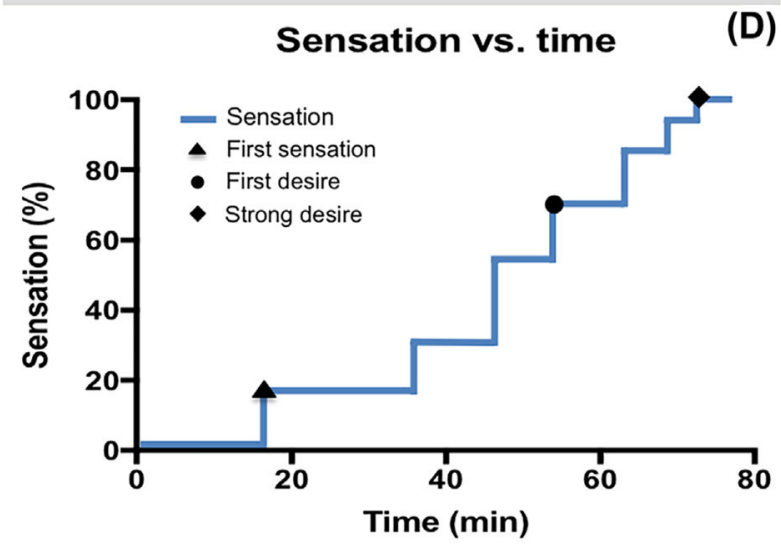

(B)
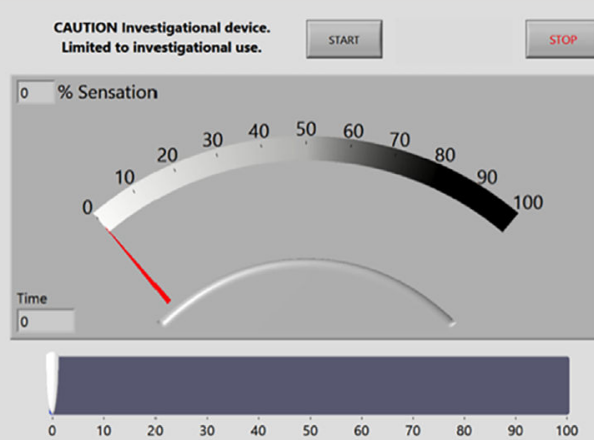

Strong

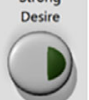

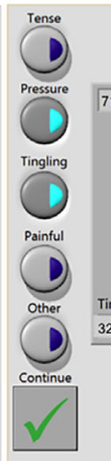

CAUTION Investigational device. Limited to investigational use.

$71 \%$ Sensation
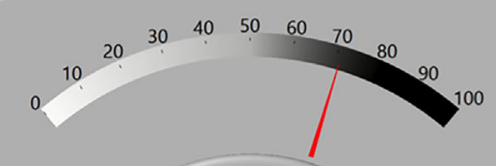

Time
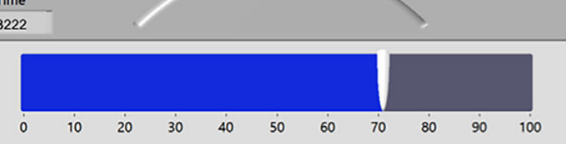

D)

\begin{tabular}{|c|c|c|c|c|c|}
\hline $\begin{array}{l}\text { Event } \\
\#\end{array}$ & $\begin{array}{l}\text { Time } \\
(\min )\end{array}$ & $\begin{array}{l}\text { Sens. } \\
(\%)\end{array}$ & $\begin{array}{l}\text { Vol } \\
(\mathrm{ml})\end{array}$ & VST & Description(s) \\
\hline 1 & 16.8 & 17 & 183 & FS & Tingling \\
\hline 2 & 35.9 & 31 & 391 & & Tingling \\
\hline 3 & 46.5 & 54 & 506 & & Tingling \\
\hline 4 & 53.7 & 71 & 585 & FD & Pressure-tingling \\
\hline 5 & 63.3 & 85 & 690 & & Pressure-tingling \\
\hline 6 & 68.8 & 94 & 750 & & None \\
\hline 7 & 72.7 & 100 & 791 & SD & $\begin{array}{l}\text { Tense-pressure- } \\
\text { tingling }\end{array}$ \\
\hline
\end{tabular}

FIGURE 1.

A, Screenshot of sensation meter with associated Start and Stop buttons, slider bar interface (bottom), and verbal sensation threshold (VST) selection (right). B, Screenshot of sensation meter after change in sensation from example participant data (see Figure 1D). At $53.7 \mathrm{~min}$ (3222 $\mathrm{s}$ as measured by sensation meter), the participant perceived bladder sensation at $71 \%$ (bottom), selected first desire from the VST selection (right), and marked pressure and tingling from the descriptors selection (left). C, Same participant's sensation meter data plotted as \% sensation versus time, with associated VSTs marked accordingly. D, Chart corresponding to Figures 1B and 1C shows that participant perceived seven sensation events (Column 1) at the times provided (Column 2). The corresponding \% sensation (Column 3), estimated filling volume (Column 4), VSTs: first sensation (FS), first desire (FD), and strong desire (SD) (Column 5), and sensation descriptors (Column 6) are also recorded 
(A) Sensation events perfill

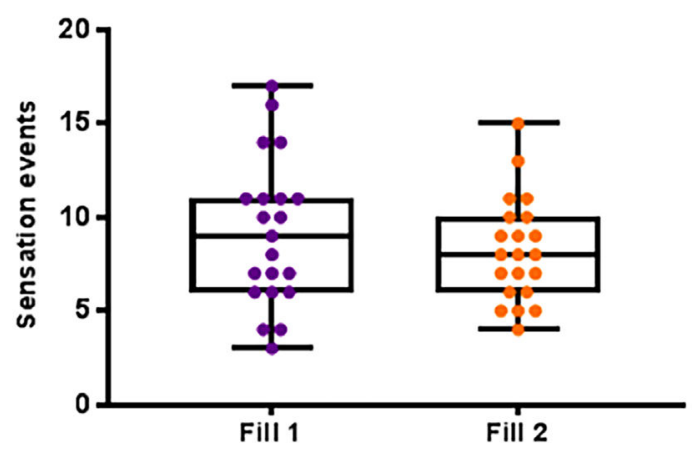

(B) Average duration per sensation event

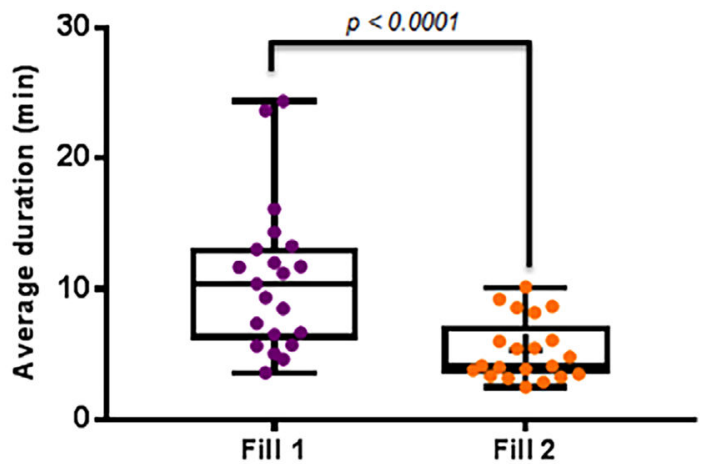

(C) Average volume per sensation event

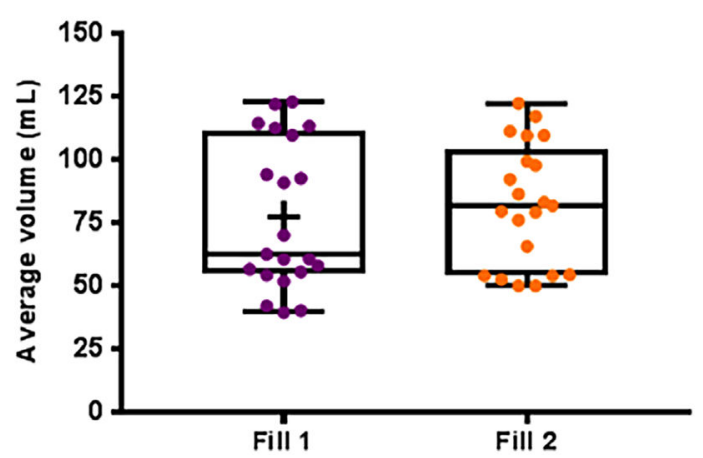

FIGURE 2.

Box plots of sensation event changes between fills. Each dot represents one participant, (total: $n=21$ ) with fill 1 (purple) versus fill 2 (orange). A, Number of sensation events. B, Average duration between sensation events, $P<0.0001$ between fill 1 and fill 2. C, Average volume per sensation event 


\section{(A) Sensation events per capacity quartile Fill 1}

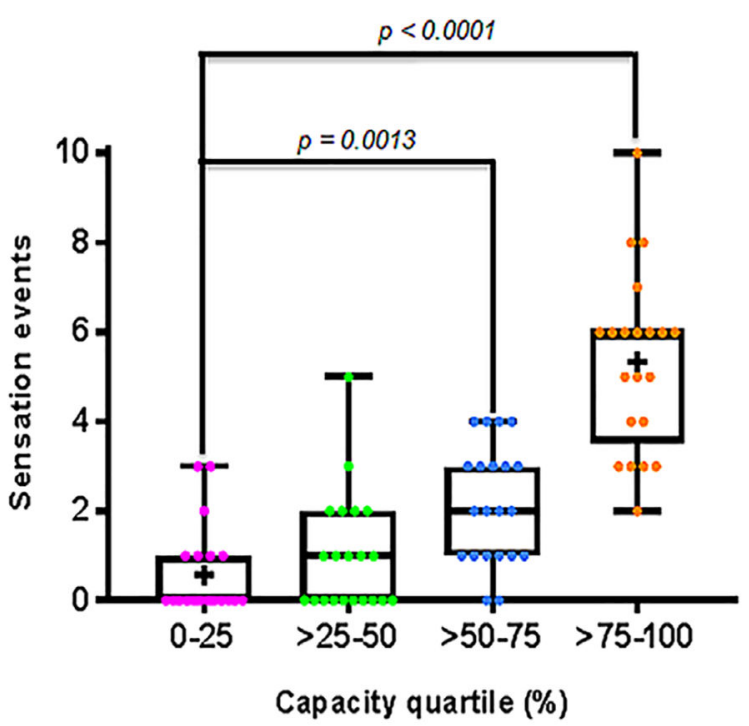

(B)

\section{Sensation events per capacity quartile Fill 2}

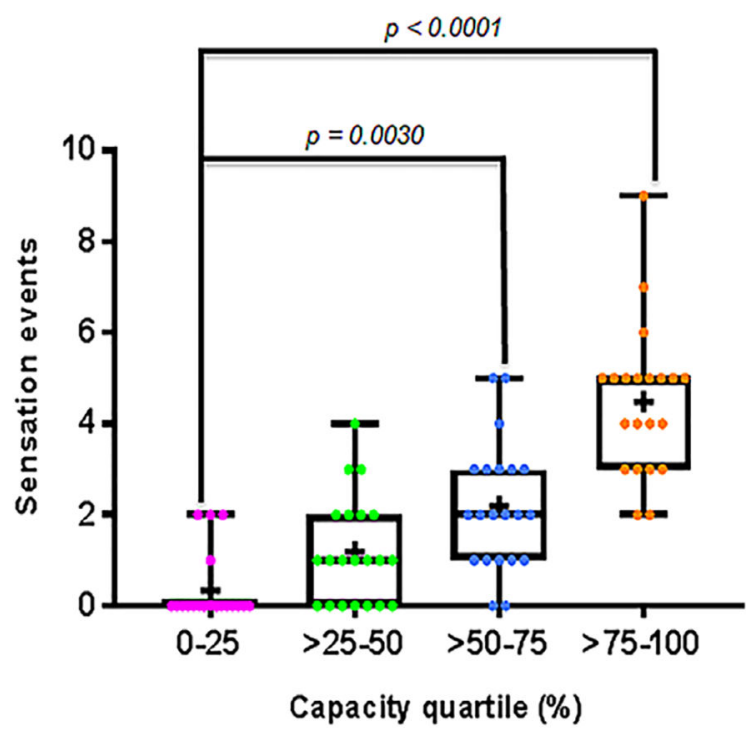

FIG. 3.

Changes in sensation events throughout the course of filling. Number of sensation events per capacity quartile (A, fill 1 and B, fill 2). Each dot represents one participant (total: $n=21$ ). Capacity quartiles are marked pink (0-25), green $(>25-50)$, blue $(>50-75)$, and orange $(>75-100)$. Both fills demonstrate a significant difference in the number of sensation events between the first and third (A, $P=0.013$ and B, $P=0.003)$ and the first and final $(\mathrm{A}, P<$ 0.0001 and $\mathrm{B}, P<0.0001)$ capacity quartiles 
(A)

Descriptors vs. \% Sensation Fill 1

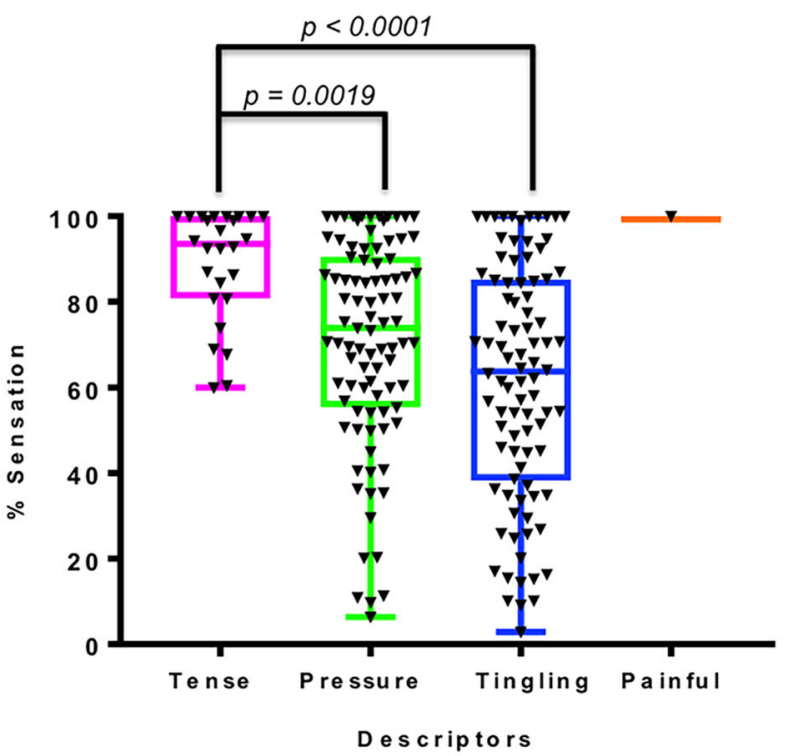

(B)

Descriptors vs. \% Sensation Fill 2

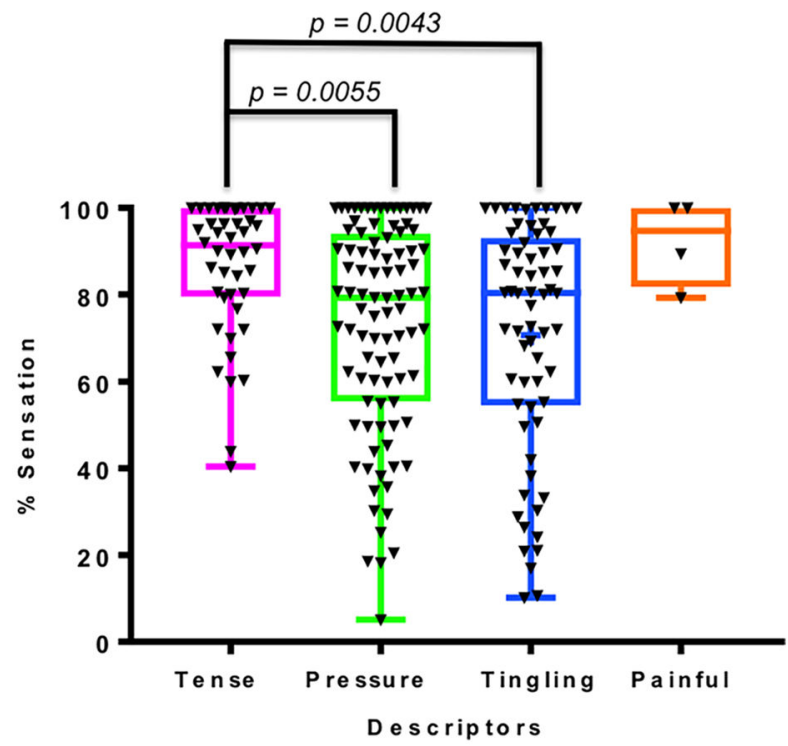

FIG. 4.

Descriptors and the \% sensation at which they were selected (A, fill 1 and B, fill 2, $n=18$ ).

Each mark (black triangle) represents one event where a specific descriptor was selected.

Descriptors are classified as pink (tense), green (pressure), blue (tingling), and orange (painful). Both fills had a significant difference in the frequency of descriptor selection between tense and pressure (A, $P=0.0019$ and $\mathrm{B}, P=0.0055)$ and tense and tingling $(\mathrm{A}, P<$ 0.0001 and $\mathrm{B}, P=0.0043$ ) 